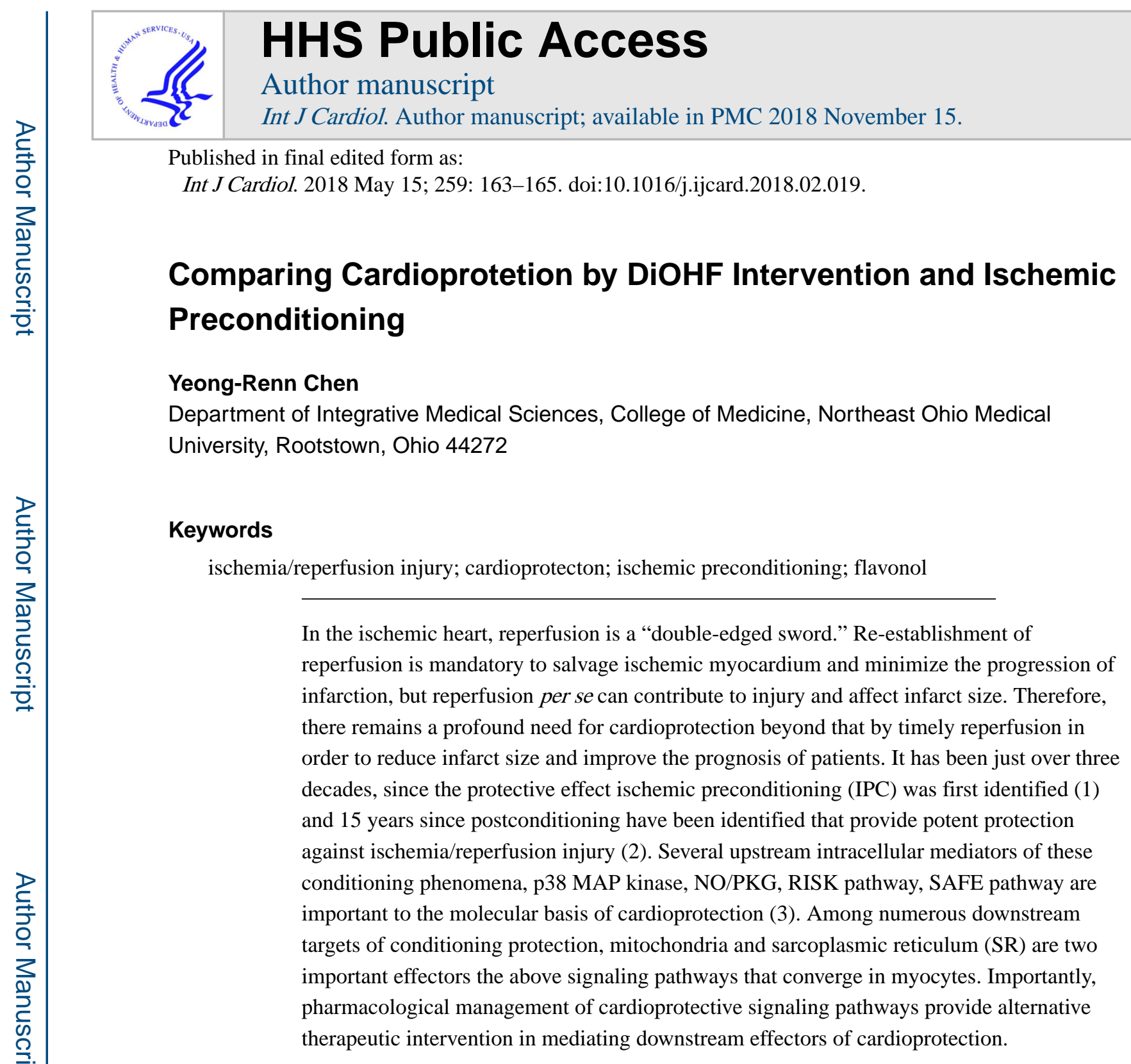

\title{
Signaling involved in SR
}

Now, Woodman and co-workers report that antioxidant flavonol can mitigate reperfusion injury via regulating the signaling pathway involved in inhibition of phospholamban (PLN) and JNK2 (4). Cytosolic calcium overload during early reperfusion contributes to calcium oscillation and eventual uncoordinated contracture, MPTP opening, and eventual cell death. $\mathrm{SR}$ is thus a potential target of protection during early reperfusion. IPC has been reported to improve SR function via enhancing calcium uptake and release along with increased CaMKmediated phosphorylation of Ryanodine receptor, SERCA, and PLN at the end of reperfusion (5). However, reperfusion-induced ROS overproduction also stimulates excess CaMKII-mediated PLN activation, leading to SR calcium overloading that contributes to

\footnotetext{
Address correspondence to: Professor Yeong-Renn Chen, Department of Integrative Medical Sciences, College of Medicine, Northeast Ohio Medical University, 4209 State Route 44, PO Box 95, Rootstown, OH 44272, USA, Tel.: 614-325-6537, Fax: 614-325-5978, ychen1@neomed.edu.

Conflicts of Interest: YRC has no conflicts of interest.
} 
myocyte injury. Woodman's group report a similar mechanism of correcting intracellular calcium handling by pharmacological management with a specific flavonoid, $3^{\prime}, 4^{\prime}$ dihydroxyflavonol (DiOHF). Besides the antioxidant function of suppressing oxidative stress, current study further concludes DiOHF affects SR function by alleviating excess CaMKII activation during early reperfusion. By in vitro biochemical analysis, DiOHF reduces CaMKII over-activation by competing with the ATP-binding site of kinase. Binding of DiOHF further down-regulates CaMKII activity by changing the conformation of $\mathrm{Ca}^{2+}$ / calmodulin-binding site. The above inhibitory mechanism might potentially up-regulate cellular bioavailability of ATP for other upstream intracellular signaling pathways of cardioprotection.

\section{Signaling involved in Mitochondria}

Tethering or connection between smooth ER and mitochondria in myocytes facilitates interaction of SR with MPTP. SR calcium overloading and calcium oscillation during reperfusion contribute to MPTP opening. Substantial evidence has supported mitochondria, where most of the above signaling pathways converge, are important effectors of flavonol protection. These pathways involve SAFE, RISK, and p38MAPK, and share similar mechanisms to those of pre- and post-conditioning protection. Beyond antioxidant function, DiOHF preserves mitochondrial function during early reperfusion, and conserves ATP bioavailability for upstream of signaling pathways.

Perhaps even more important is the development of an effective competitive inhibitor capable to suppress the kinase activation controlling the upstream of injurious signaling pathways (e.g. specific isoform of MAP kinase) and promote kinase activation mediating protective signaling pathways (e.g. RISK and SAFE).

\section{Signaling involved in JNKs downregulation}

JNKs belongs to the superfamily of MAP-kinases indispensable for both cell proliferation and apoptosis. Reperfusion injury is mediated in part by activation of stress kinase JNK $(6,7)$. The redox stress of excess ROS during reperfusion stimulates activation of JNKs via phosphorylation by two distinct MAP kinase kinases (MKK4/7), which in turn promote apoptotic signaling via two distinct mechanisms involved in nucleus- or mitochondriatargeted events. In the pathway directed at mitochondria, activated JNKs translocate to mitochondria, where JNKs can phosphorylate the Bcl2 proteins to antagonize the antiapoptotic activity Bcl2. In addition, JNK can stimulate the release of cytochrome $c$ from mitochondria through a Bid-Bax-dependent mechanism, promoting apoptosomes formation and the activation of caspase cascade. JNK is activated by ischemia/reperfusion but not during ischemia alone. The role of JNKs in the venue of IPC has been controversial with studies, suggesting both a protective and detrimental aspect to JNKs activation (7). The current study supports pharmacological management with DiOHF down-regulates JNKs activation to exacerbate protective effect of reperfusion injury. However, no further evidence was provided to bridge the knowledge gaps of how DiOHF regulates upstream MKK4/7 function and downstream Bcl2-associated pro-apoptotic signaling, as well as mitochondrial localization of activated JNKs. 


\section{Perspectives}

Besides the effect of excess production of ROS, reperfusion mediates overshooting of the myocardial $\mathrm{pO}_{2}$, which is correlated to decreased mitochondrial function, redox dysfunction, and enhanced disease marker of oxidative posttranslational modifications of mitochondrial proteins in the postischemic heart (8). The underlying mechanisms of how these hyperoxic conditions contribute to reperfusion injury and eventual cell death via promoting detrimental signaling pathways or down-regulation of protective signaling pathways are not fully understood. However, experimental evidence unequivocally supports that IPC attenuates reperfusion-mediated tissue hyperoxia and improves mitochondrial function in the myocardium of the mouse model. L-band EPR further indicates IPC corrects associated redox dysfunction induced by reperfusion in the mouse heart (9). No substantial evidence has been shown yet supporting that IPC can protect the myocardial proteins from detrimental oxidative modifications by reperfusion. Significance of the above IPC protection raises several questions for future investigation. Could antioxidant flavonol exert protection via modulation tissue $\mathrm{pO}_{2}$ and associated redox biology in heart?

Finally, the proposed mechanism concluded from current investigation reinforces a seminal role of mitochondria as the effector of cardioprotection by pharmacological interventions, directly or indirectly. This raises the other question for future investigation. Could DiOHF benefit myocardial blood flow via enhancing metabolic dilation controlled by mitochondria? In murine models, transgenic overexpression of human SOD2 induces super-normal cardiac function by enhancing mitochondrial function and metabolic dilation (10). Delayed preconditioning can also upregulate SOD2 and reduce ROS, but it is unclear if the conditioning phenomena can be translated to promoting metabolic dilation.

Is the cardiopretective story of DiOHF finished? Most definitely not. The nature of DiOHF interaction with mitochondria, the pathways of modulating the $\mathrm{pO}_{2}$ in myocardium, and metabolic dilation-based protective mechanism remain to be explored. Apart from the translational gap from rodent to human, we need to know more about mechanistic insights. The fun is just beginning.

\section{Acknowledgments}

Author thanks Professor William M. Chilian (Northeast Ohio Medical University, Rootstown, Ohio, USA) for critical review. Funding source: HL083237 from National Institutes of Health (NIH).

\section{References}

1. Murry CE, Jennings RB, Reimer KA. Preconditioning with ischemia: a delay of lethal cell injury in ischemic myocardium. Circulation. 1986; 74:1124-1136. [PubMed: 3769170]

2. Zhao ZQ, Corvera JS, Halkos ME, Kerendi F, Wang NP, Guyton RA, Vinten-Johansen J. Inhibition of myocardial injury by ischemic postconditioning during reperfusion: comparison with ischemic preconditioning. American journal of physiology. Heart and circulatory physiology. 2003; 285:H579-588. [PubMed: 12860564]

3. Heusch G. Molecular basis of cardioprotection: signal transduction in ischemic pre-, post-, and remote conditioning. Circulation research. 2015; 116:674-699. [PubMed: 25677517] 
4. Chin KY, Silva LS, Darby IA, Ng DVH, Woodman OL. Protection against reperfusion injury by $3^{\prime}$, $4^{\prime}$-dihydroxyflavonol in rat isolated hearts invovles inhibition of phospholamban and JNK. Intenational Journal of Cardiology. 2018; 254:265-271.

5. Osada M, Netticadan T, Tamura K, Dhalla NS. Modification of ischemia-reperfusion-induced changes in cardiac sarcoplasmic reticulum by preconditioning. The American journal of physiology. 1998; 274:H2025-2034. [PubMed: 9841529]

6. Yin T, Sandhu G, Wolfgang CD, Burrier A, Webb RL, Rigel DF, Hai T, Whelan J. Tissue-specific pattern of stress kinase activation in ischemic/reperfused heart and kidney. The Journal of biological chemistry. 1997; 272:19943-19950. [PubMed: 9242662]

7. Hausenloy DJ, Yellon DM. Survival kinases in ischemic preconditioning and postconditioning. Cardiovascular research. 2006; 70:240-253. [PubMed: 16545352]

8. Zhao X, He G, Chen YR, Pandian RP, Kuppusamy P, Zweier JL. Endothelium-derived nitric oxide regulates postischemic myocardial oxygenation and oxygen consumption by modulation of mitochondrial electron transport. Circulation. 2005; 111:2966-2972. [PubMed: 15939832]

9. Zhu X, Liu B, Zhou S, Chen YR, Deng Y, Zweier JL, He G. Ischemic preconditioning prevents in vivo hyperoxygenation in postischemic myocardium with preservation of mitochondrial oxygen consumption. American journal of physiology. Heart and circulatory physiology. 2007; 293:H14421450. [PubMed: 17513495]

10. Kang PT, Chen CL, Ohanyan V, Luther DJ, Meszaros JG, Chilian WM, Chen YR. Overexpressing superoxide dismutase 2 induces a supernormal cardiac function by enhancing redox-dependent mitochondrial function and metabolic dilation. Journal of molecular and cellular cardiology. 2015; 88:14-28. [PubMed: 26374996] 




ATP $\stackrel{\oplus}{\longrightarrow}$ RISK pathway $\stackrel{\oplus}{\longrightarrow}$
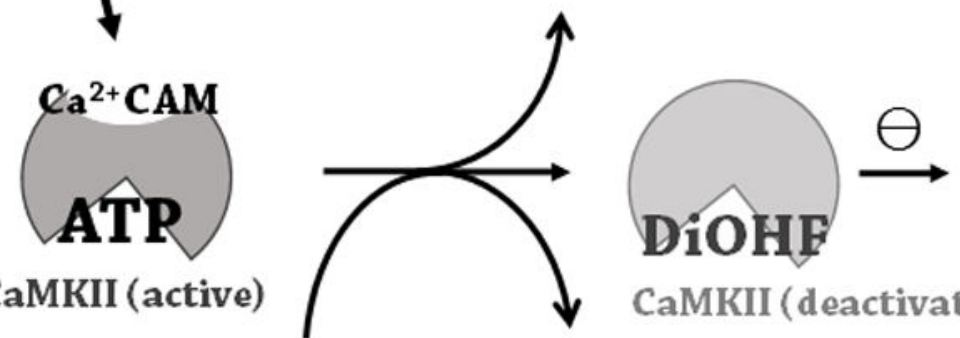

DiOHE

$\mathrm{Ca}^{2+}$ overloading

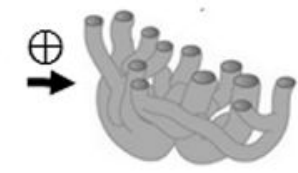

CaMKII (deactivated)

DiOHF ATP $\stackrel{\oplus}{\longrightarrow}$ SAFE pathway $\stackrel{\oplus}{\longrightarrow}$

Figure.

Interaction of DiOHF with CaMKII in cardioprotection. Antioxidant flavonol, DiOHF, reduces ischemia/reperfusion (I/R)-induced CaMKII over-activation by competing ATPbinding site of the kinase, which alleviates I/R-mediated calcium overloading/calcium oscillation and improves SR function. Competitive inhibition of CaMKII by DiOHF further up-regulates intracellular bioavailability of ATP for upstream protective pathways of SAFE and RISK, which subsequently promotes the effector of mitochondria for cardioprotection against reperfusion injury. 